\title{
Effectiveness of a Physical Activity and Weight Loss Intervention for Middle-Aged Women: Healthy Bodies, Healthy Hearts Randomized Trial
}

\author{
Molly B. Conroy, MD, MPH' , Kathleen L. Sward, PhD, MPH ${ }^{2}$, Kathleen C. Spadaro, PhD ${ }^{3}$, \\ Dana Tudorascu, $P h D^{7}$, Irina Karpov, $M S^{7}$, Bobby L. Jones, $P h D^{7}$, Andrea M. Kriska, $P h D^{7}$, and \\ Wishwa N. Kapoor, MD, MPH
}

'University of Pittsburgh, Pittsburgh, PA, USA; ${ }^{2}$ HeartChange http://www. drkathysward.com; ${ }^{3}$ Chatham University, Pittsburgh, PA, USA.

\begin{abstract}
BACKGROUND: Physical inactivity is a significant risk factor for cardiovascular disease and remains highly prevalent in middle-aged women.

OBJECTIVE: We hypothesized that an interventionist-led (IL), primary-care-based physical activity (PA) and weight loss intervention would increase PA levels and decrease weight to a greater degree than a self-guided (SG) program.
\end{abstract}

DESIGN: We conducted a randomized trial.

PARTICIPANTS: Ninety-nine inactive women aged 45-65 years and with BMI $\geq 25 \mathrm{~kg} / \mathrm{m}^{2}$ were recruited from three primary care clinics.

INTERVENTIONS: The interventionist-led (IL) group $(n=49)$ had 12 weekly sessions of 30 min discussions with 30 min of moderate-intensity PA. The self-guided (SG) group $(n=50)$ received a manual for independent use.

MAIN MEASURES: Assessments were conducted at 0, 3, and 12 months; PA and weight were primary outcomes. Weight was measured with a standardized protocol. Leisure PA levels were assessed using the Modifiable Activity Questionnaire. Differences in changes by group were analyzed with a t-test or Wilcoxon rank-sum test. Mixed models were used to analyze differences in changes of outcomes by group, using an intention-to-treat principle. KEY RESULTS: Data from 98 women were available for analysis. At baseline, mean (SD) age was 53.9 (5.4) years and $37 \%$ were black. Mean weight was 92.3 (17.7) $\mathrm{kg}$ and mean BMI was 34.7 (5.9) kg/m². Median PA level was 2.8 metabolic equivalent hours per week (MET-hour/week) (IQR 0.0, 12.0). At 3 months, IL women had a significantly greater increase in PA levels (7.5 vs. 1.9 MET-hour/week; $p=0.02$ ) than SG women; there was no significant difference in weight change. At 12 months, the difference between groups was no longer significant (4.7 vs. 0.7 MET-hour/week; $p=0.38)$. Mixed model analysis showed a significant $(p=0.048)$ difference in PA change between groups at 3 months only.

Electronic supplementary material The online version of this article (doi:10.1007/s11606-014-3077-5) contains supplementary material, which is available to authorized users.

Received March 20, 2014

Revised September 8, 2014

Accepted October 4, 2014

Published online November 13, 2014
CONCLUSIONS: The IL intervention was successful in increasing the physical activity levels of obese, inactive middle-aged women in the short-term. No significant changes in weight were observed.

KEY WORDS: physical activity; exercise; clinical trial; intervention. J Gen Intern Med 30(2):207-13 DOI: $10.1007 / \mathrm{s} 11606-014-3077-5$

(c) Society of General Internal Medicine 2014

\section{INTRODUCTION}

Obesity and physical inactivity are at epidemic rates in the United States, and medical complications of obesity and physical inactivity are commonly treated in primary care settings. ${ }^{1-3}$ Women are disproportionately affected by obesity ${ }^{4,5}$ and physical inactivity, and have more primary care encounters than men. ${ }^{6}$ Higher levels of physical activity (PA) are associated with a lower prevalence of obesity, and PA also has an independent beneficial effect on numerous health outcomes important to women (e.g., cardiovascular disease, breast cancer, depression). ${ }^{7}$ Therefore, there is both a great need and an opportunity to develop primary care-based interventions tailored for women that address physical activity and weight.

Previous studies of primary care interventions to address obesity and physical activity have had null or mixed results, leading the United States Preventive Services Task Force (USPSTF) to assign a grade of "I" (or inconclusive) to both low-intensity and moderate-intensity weight loss interventions and physical activity interventions delivered in primary care settings. ${ }^{8}$ Only more intensive weight loss interventions, which typically include a higher number of sessions and behavioral principles such as self-monitoring and goalsetting, are supported by sufficient evidence to earn a USPSTF grade of "B" (i.e., a recommended service with moderate to high certainty of benefit). ${ }^{9}$

The majority of primary care interventions conducted to date have not focused primarily on women. Women may have different needs, due to a higher number of lifetime weight loss 
attempts $^{10}$ and lower baseline physical activity levels ${ }^{3}$ than men. Middle-age is an especially relevant intervention period for women, as physical activity levels tend to decrease, whereas weight and cardiovascular disease risk increase. ${ }^{11,12}$ Previous lifestyle interventions targeting women tended to be conducted in community samples that may be healthier on average than patients in primary care settings. 13,14

We conducted a randomized, controlled trial of a PA and weight loss intervention for overweight and obese, inactive women, delivered in coordination with primary care. We hypothesized that an interventionist-led (IL), primary carebased PA and weight loss intervention would increase PA levels and decrease weight to a greater degree than a selfguided (SG) program in the short-term (3 month) and longterm (12 months). We also examined the impact of the intervention on reduction of secondary outcomes of body mass index (BMI), waist circumference, and blood pressure. We also conducted a secondary analysis to determine if the effects of the intervention varied by race.

\section{METHODS}

\section{Design}

The Healthy Bodies Healthy Hearts (HBHH) study was a randomized, controlled trial delivered in coordination with primary care and intended to increase leisure physical activity (PA) levels and decrease weight in overweight and obese, inactive, middle-aged women. Primary outcomes were changes in PA level and weight at 3 and 12 months. Secondary outcomes were BMI, waist circumference, and blood pressure. The protocol was approved by University of Pittsburgh institutional review board (IRB) and registered with ClinicalTrials.gov (NCT01099007).

\section{Setting}

Assessments and the interventionist-led group intervention were conducted at the largest and most centrally located of three primary care practices affiliated with University of Pittsburgh Medical Center (UPMC). Two of the primary care practices were general medicine academic practices with faculty and resident providers; the last was a primary care practice for mid-life women with both obstetrics and gynecology (OB-GYN) and general medicine faculty providers.

\section{Recruitment and Baseline Screening}

Recruitment began in June 2009 and was completed in February, 2010. We recruited women aged $45-65$ years, with a BMI $\geq 25 \mathrm{~kg} / \mathrm{m}^{2}$ who were relatively physically inactive (less than one hour of PA per week), and receiving primary care at one of three UPMC practices. Exclusions included: unstable cardiac or pulmonary disease (e.g., recent myocardial infarction), poorly controlled hypertension (i.e., systolic blood pressure $[\mathrm{SBP}] \geq 180$ ), primary care physician (PCP) unwilling to allow moderate PA, and participant unable to perform moderate PA (i.e., unable to walk due to severe pain).

Our primary recruitment tool was a best-practice alert in the electronic health record that notified the primary care PCP of a woman who potentially qualified for the study. If the woman expressed interest, the PCP would enter a referral order and endorse that moderate PA was safe for the patient. After referral, a screening call was made to the woman by study staff to assess eligibility and interest. If the woman met criteria and was interested, a 60-90 min, in-person baseline assessment was scheduled.

Baseline screening started with a review of the consent form and the participation requirements of the study by study staff. Following the signed consent, height, weight, blood pressure, and waist circumference were obtained by trained study personnel. The Modified Activity Questionnaire (MAQ) was administered by a trained research staff member. ${ }^{15}$

\section{Randomization}

Once the participant had been screened, the consent signed, and the initial baseline assessments completed, randomization occurred in a 1:1 allocation. Each woman was allowed to draw a sealed envelope that contained a designation assignment, either interventionist-led (IL) or self-guided (SG).

\section{Interventions}

IL groups were comprised of 12 weekly group sessions and were conducted in a conference room in the largest of the three primary care practices. There were 12 designated topics for the sessions (Online Appendix Table A), led by the study physician (MBC), who was also a provider at the practice, or one of two PhD-level interventionists (KLS, KCS) not affiliated with the practice. The content focused on physical activity, diet, and stress relief adapted from a lifestyle intervention for premenopausal and postmenopausal women ${ }^{13,16,17}$ as well as portions of the Diabetes Prevention Program (DPP). ${ }^{18}$ Participants were given PA and dietary goals similar to those given in the DPP, with target calorie and fat gram goals based on starting weight and a PA goal of at least 150 min of moderate PA per week. Participants were given a calorie-counter book, a pedometer, and sheets for tracking PA and diet; tracking sheets were not collected or reviewed by the interventionist. The sessions included $30 \mathrm{~min}$ discussions followed by 30 min of group-based, moderate-intensity PA.

Mindfulness concepts were formally introduced through mindful eating and mindful PA sessions, and were integrated throughout the 12 week intervention. ${ }^{19}$ The participants practiced bringing their awareness and attention to the present moment with intention and without judgment of thoughts, 
emotions, body sensations, sensory perceptions, and behavioral actions. They were instructed to be an impartial witness to themselves during the experience of eating and exercise.

SG participants received a 12-week, self-guided manual based on the American Heart Association's Choose to Move program $^{20}$ at randomization, as well as a calorie-counter book and a pedometer. There was no interventionist contact with the SG group, but they had the contact information of study personnel for any questions or concerns.

\section{Outcome Assessments/Measures}

In-person assessments for outcomes were performed at 3 and 12 months after baseline. Participants were not compensated for the 3-month visit, but did receive a \$25 payment for completing the 12-month visit either in person or over the phone. If it was not possible for a participant to come in for a 12-month, in-person assessment, a brief version phone visit was conducted, focusing on PA data and a self-reported weight. Referring providers received letters after the 3 and 12 month outcome assessment visits informing them of their patients' current weights and physical activity statuses.

\section{Primary Outcomes}

Weight was measured by a trained staff member in clinic using a standard balance beam scale (SECA Medichoice) and following a written protocol. ${ }^{13}$

Physical Activity was measured using the one-month version of the Modifiable Activity Questionnaire (MAQ), administered by a trained, certified staff member. We focused on leisure activity estimates, because our intervention was designed to target leisure PA. ${ }^{13}$ PA levels were calculated as the product of the duration and frequency of each activity (in hours per week), weighted by an estimate of the metabolic equivalent (MET) of that activity and summed for all activities performed. PA data were expressed as metabolic equivalent hours per week (MET-hour/week). The MAQ has been shown to be both reliable and valid, and has been widely used in numerous populations and intervention studies. $^{15,18}$

\section{Secondary Outcomes}

Clinically relevant secondary outcomes, typically lowered by a physical activity and weight loss intervention, include:

BMI, calculated using a standard equation.

Waist Circumference, measured using a non-stretch tape measure marked in centimeters.

Blood pressure, measured averaging three readings collected using a written protocol ${ }^{13}$ by trained staff using manual blood pressure cuffs (Welch Allyn).

\section{Exploratory Outcomes}

Psychosocial outcomes included Physical Activity Enjoyment Scale (PACES), ${ }^{21}$ Beck Depression Inventory (BDI), ${ }^{22}$ Pittsburgh Sleep Quality Index (PSQI), ${ }^{23}$ Short Form-12 Health Survey (SF-12), ${ }^{24}$ and Exercise Self-Efficacy. ${ }^{25-27}$ Information about these measures and the results of these analyses are found in the online Exploratory Psychosocial Measures Appendix (Online Appendix B; Online Appendix Tables B1/B2).

\section{Other Variables}

Race, education, marital status, and medical comorbidities were assessed using self-report. The first three were assessed at baseline, and the last at 12 months $(n=83)$.

\section{Statistical Methods}

Descriptive statistics characterized the outcome variables at baseline, 3 months and 12 months. To assess differences between groups at baseline and 3-month and baseline and 12-month follow-up, two sample t-tests (continuous, normally distributed outcomes) or Wilcoxon's Rank Sum (continuous, non-normally distributed) were conducted. Our primary outcome analysis compared groups according to the intention-totreat approach. We used mixed models with fixed effects of group, time ( 3 and 12 months) and group $\mathrm{x}$ time interaction, controlling for baseline measure and BMI for primary outcomes and only for baseline measurements for secondary outcomes. For models, square root transformation was used for the physical activity outcome variable. The mixed models take into account the correlation among measurements within a person over time. We also conducted analyses stratified by race. Analyses were conducted using SAS version 9.3 statistical software (SAS Institute Inc., Cary, NC, USA).

\section{RESULTS}

\section{Enrollment, Follow-up, and Population Characteristics}

We assessed a total of 182 women for enrollment in our study (Fig. 1). We excluded 83 women who did not meet inclusion criteria $(n=56)$ or were not interested $(n=25)$. We randomized 49 women to the interventionist-led intervention and 50 women to the self-guided intervention. Overall, we had $76 \%$ follow-up at 3 months and $86 \%$ at 12 months. For the 12-month followup, $62(74 \%)$ of 84 participating women had an in-person assessment (with study-measured weight), with the remainder of the outcomes assessed by phone. Follow-up was better in the IL group (90\% at 3 months and $96 \%$ at 12 months) than in the SG group (63\% at 3 months and $76 \%$ at 12 months), but otherwise did not differ by other participant characteristics. 


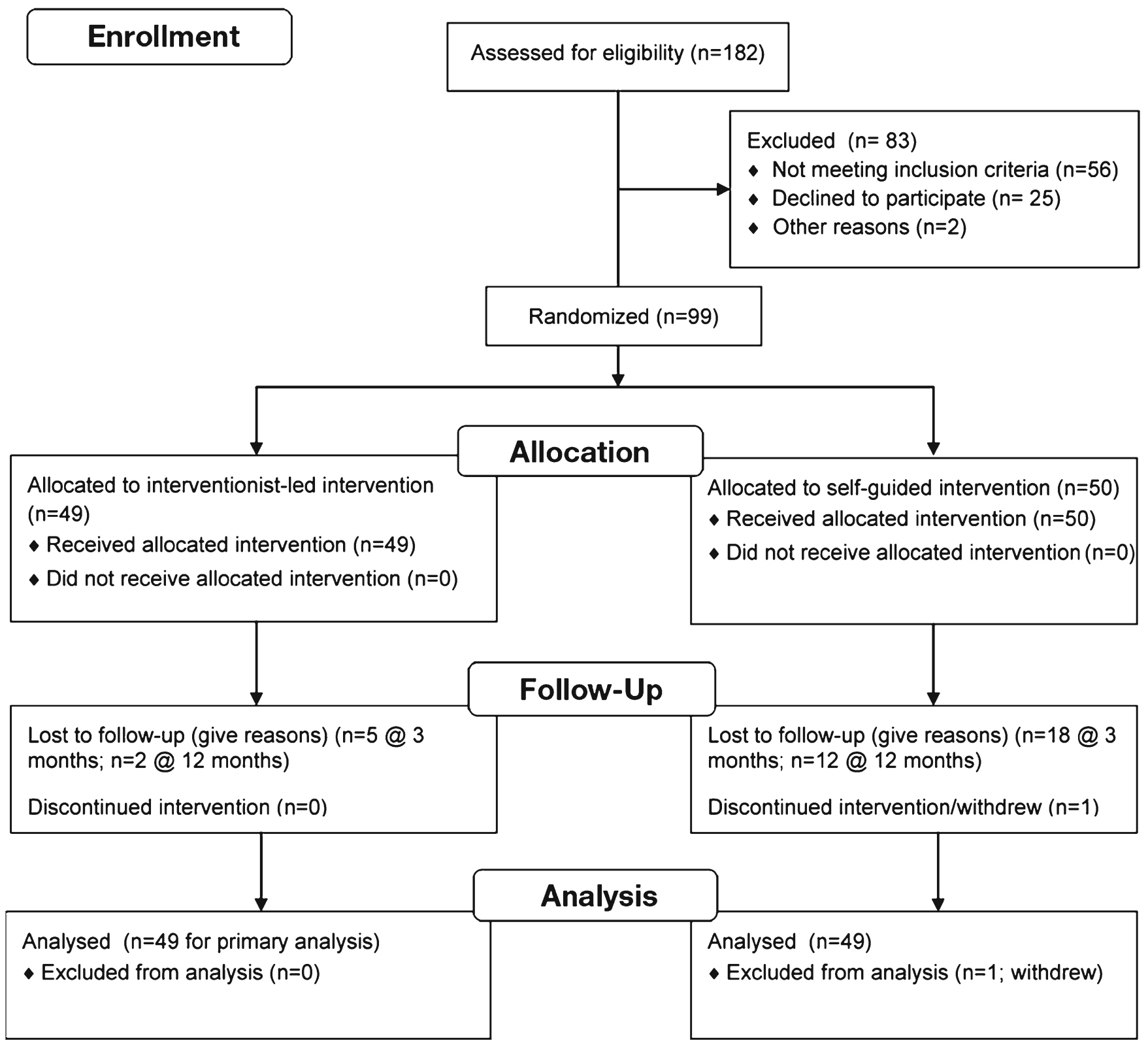

Figure 1. Healthy bodies, healthy hearts flow diagram.

For the 49 women in the IL intervention, 17 (35\%) attended nine or more of the sessions (75\% adherence); 30 (61\%) attended at least six of the sessions (50\% adherence).

The baseline characteristics of the $\mathrm{HBHH}$ participants are listed in Table 1. The average age of the group was 53.9 (5.4) years and the average BMI was 34.7 (5.9). The mean SBP was in the normotensive range (118.3 (14.6). Women were relatively sedentary with a median PA level of 2.8 (IQR 0, 12.0) MET-hour/week. Most characteristics did not differ by randomized group, although women in the IL group had a higher BMI (36.1 vs. 33.4; $p=0.02$ ) when compared to the SG group.

Medical comorbidities were common in our population, particularly high blood pressure (56\%), arthritis
(48\%), and depression (40\%), diabetes (23\%), and sleep apnea $(24 \%)$.

\section{Changes in Primary Outcomes at 3 months}

At 3 months, women in the interventionist-led intervention reported a median increase in PA of 7.5 MET-hour/ week, which was significantly greater that the 1.9 METhour/week increase in the self-guided group $(p=0.02$; Table 2). Weight loss in both groups was quite modest (mean loss $-1.4 \mathrm{~kg}$ ), and there was no significant difference between changes in the IL and SG groups (-1.7 vs. -1.1 ; $p=0.41$; Table 2). 
Table 1. Baseline Characteristics of HBHH Participants $(n=98)$

\begin{tabular}{|c|c|c|c|c|}
\hline Variable & Total $(n=98)$ mean(std) / n(\%) & IL $(n=49)$ mean(std) / n(\%) & SG (n=49) mean(std) / n(\%) & p value \\
\hline Age & $53.9(5.4)$ & $53.8(5.3)$ & $54.0(5.6)$ & 0.83 \\
\hline Race & & & & 0.84 \\
\hline White & $59(60.2 \%)$ & $31(63.3 \%)$ & $28(57.1 \%)$ & \\
\hline AA & $36(36.7 \%)$ & $17(34.7 \%)$ & $19(38.8 \%)$ & \\
\hline Asian & $1(1.0 \%)$ & $0(0.0 \%)$ & $1(2.0 \%)$ & \\
\hline Other & $2(2.0 \%)$ & $1(2.0 \%)$ & $1(2.0 \%)$ & \\
\hline Education & & & & 0.18 \\
\hline High school & $22(22.4 \%)$ & $10(20.4 \%)$ & $12(24.5 \%)$ & \\
\hline College & $35(35.7 \%)$ & $18(36.7 \%)$ & $17(34.7 \%)$ & \\
\hline Post graduate & $29(29.6 \%)$ & $18(36.7 \%)$ & $11(22.4 \%)$ & \\
\hline Other & $12(12.2 \%)$ & $3(6.1 \%)$ & $9(18.4 \%)$ & \\
\hline Marital status & & & & 0.32 \\
\hline Married & $51(52.6 \%)$ & $24(49.0 \%)$ & $27(56.3 \%)$ & \\
\hline Separated & $5(5.2 \%)$ & $2(4.1 \%)$ & $3(6.3 \%)$ & \\
\hline Divorced & $15(15.5 \%)$ & $11(22.4 \%)$ & $4(8.3 \%)$ & \\
\hline Widowed & $8(8.2 \%)$ & $5(10.2 \%)$ & $3(6.3 \%)$ & \\
\hline Never married & $17(17.5 \%)$ & $7(14.3 \%)$ & $10(20.8 \%)$ & \\
\hline Other & $1(1.0 \%)$ & $0(0.0 \%)$ & $1(2.1 \%)$ & \\
\hline PA level (MET-hour/week) ${ }^{\mathrm{a}}$ & $2.8(0,12.0)$ & $2.7(0,14.00)$ & $3.5(0.21,10.50)$ & $0.93^{*}$ \\
\hline Weight $(\mathrm{kg})$ & $92.3(17.7)$ & $95.0(18.8)$ & $89.6(16.3)$ & 0.13 \\
\hline BMI $\left(\mathrm{kg} / \mathrm{m}^{2}\right)$ & $34.7(5.9)$ & $36.1(6.0)$ & $33.4(5.4)$ & $0.02^{\dagger}$ \\
\hline Waist Circumference (cm) & 105.7 (11.4) & $106.4(12.3)$ & $105.0(10.4)$ & 0.57 \\
\hline SBP (mmHg) & $118.3(14.6)$ & $116.2(15.6)$ & $120.4(13.4)$ & 0.16 \\
\hline DBP (mmHg) & $75.2(10.3)$ & $74.9(9.5)$ & $75.5(11.2)$ & 0.79 \\
\hline
\end{tabular}

IL interventionist-led, SG self-guided

${ }^{a} P A$ data is shown as median (IQR)

"p values were calculated using Wilcoxon's Rank Sum non-parametric test

'Significant $p$ value

\section{Changes in Primary Outcomes at 12 months}

At 12 months, women in the interventionist-led intervention reported a median increase in PA of 4.7 MET-hour/week over baseline, which was no longer significantly greater that the 0.7 increase in the self-guided group ( $p=0.38$; Table 2). Modest weight loss in both groups persisted at 12 months (mean loss $-1.4 \mathrm{~kg})$, and was nearly identical between the two groups $(p=0.98$; Table 2).

\section{Changes in Secondary Outcomes at 3 and 12 Months}

Women in both groups experienced modest decreases in BMI and waist circumference at both 3 and 12 months, but the differences between the groups were not significant at either time point. There were also no significant differences in blood pressure changes between groups (Table 2).

Table 2. Changes in Primary and Secondary Outcomes at 3 and 12 Months (Complete Data Only)

\begin{tabular}{|c|c|c|c|c|c|c|c|c|}
\hline \multirow[t]{2}{*}{ Variable } & \multicolumn{4}{|l|}{ 3-month } & \multicolumn{4}{|l|}{ 12-month } \\
\hline & Total $(N=98)$ & IL $(n=49)$ & SG $(n=49)$ & p value & Total $(N=98)$ & IL $(n=49)$ & SG $(n=49)$ & p value \\
\hline \multicolumn{9}{|l|}{ Primary outcomes } \\
\hline PA level (MET-hour/week) ${ }^{*}$ & $\begin{array}{l}5.7(0.8,12.8) \\
(n=75)\end{array}$ & $\begin{array}{l}7.5(3.6,13.3) \\
(n=44)\end{array}$ & $\begin{array}{l}1.9(-0.9,6.8) \\
(n=31)\end{array}$ & $0.02^{*}$ & $\begin{array}{l}3.7(0.0,11.7) \\
(n=84)\end{array}$ & $\begin{array}{l}4.7(-0.1,12.9) \\
(n=47)\end{array}$ & $\begin{array}{l}0.7(-0.6,11.7) \\
(n=37)\end{array}$ & $0.38^{*}$ \\
\hline Weight $(\mathrm{kg})$ & $\begin{array}{l}-1.4(3.5) \\
(n=72)\end{array}$ & $\begin{array}{l}-1.7(4.0) \\
(n=42)\end{array}$ & $\begin{array}{l}-1.1(2.6) \\
(n=30)\end{array}$ & 0.41 & $\begin{array}{l}-1.4(5.7) \\
(n=82)\end{array}$ & $\begin{array}{l}-1.4(6.8) \\
(n=46)\end{array}$ & $\begin{array}{l}-1.4(3.8) \\
(n=36)\end{array}$ & 0.98 \\
\hline \multicolumn{9}{|l|}{ Secondary outcomes } \\
\hline BMI $\left(\mathrm{kg} / \mathrm{m}^{2}\right)$ & $\begin{array}{l}-0.7(1.4) \\
(n=68)\end{array}$ & $\begin{array}{l}-0.9(1.6) \\
(n=41)\end{array}$ & $\begin{array}{l}-0.5(1.1) \\
(n=27)\end{array}$ & 0.23 & $\begin{array}{l}-0.5(2.4) \\
(n=60)\end{array}$ & $\begin{array}{l}-0.4(2.6) \\
(n=36)\end{array}$ & $\begin{array}{l}-0.7(2.1) \\
(n=24)\end{array}$ & 0.69 \\
\hline Waist circumference $(\mathrm{cm})$ & $\begin{array}{l}-2.5(3.9) \\
(n=68)\end{array}$ & $\begin{array}{l}-2.7(3.9) \\
(n=41)\end{array}$ & $\begin{array}{l}-2.2(4.0) \\
(n=27)\end{array}$ & 0.65 & $\begin{array}{l}-2.2(6.6) \\
(n=61)\end{array}$ & $\begin{array}{l}-1.6(6.3) \\
(n=37)\end{array}$ & $\begin{array}{l}-3.2(7.1) \\
(n=24)\end{array}$ & 0.35 \\
\hline $\mathrm{SBP}(\mathrm{mmHg})$ & $\begin{array}{l}-0.2(15.1) \\
(n=68)\end{array}$ & $\begin{array}{l}-0.8(16.3) \\
(n=41)\end{array}$ & $\begin{array}{l}0.9(15.0) \\
(n=27)\end{array}$ & 0.67 & $\begin{array}{l}4.3(14.8) \\
(n=61)\end{array}$ & $\begin{array}{l}1.6(15.7) \\
(n=37)\end{array}$ & $\begin{array}{l}8.5(12.6) \\
(n=24)\end{array}$ & 0.07 \\
\hline DBP (mmHg) & $\begin{array}{l}4.5(9.5) \\
(n=68)\end{array}$ & $\begin{array}{l}4.1(9.5) \\
(n=41)\end{array}$ & $\begin{array}{l}5.1(9.7) \\
(n=27)\end{array}$ & 0.66 & $\begin{array}{l}1.6(9.6) \\
(n=61)\end{array}$ & $\begin{array}{l}0.2(8.5) \\
(n=37)\end{array}$ & $\begin{array}{l}3.9(11.0) \\
(n=24)\end{array}$ & 0.14 \\
\hline
\end{tabular}

Participants who had missing data at either 3 months or 12 months were assumed to have no change from last measured value (i.e., last observation carried forward method)

IL interventionist-led, SG self-guided

"Data are median (IQR); $p$ values were calculated using Wilcoxon's Rank Sum non-parametric test 


\section{Results Stratified by Race}

We also performed results stratified by race; these were similar to overall study results. There was a greater increase in physical activity in both white and non-white women in the IL group when compared to SG women, with results reaching statistical significance in white women at 3 months (data not shown).

\section{Results of Linear Mixed Effects Model}

Primary outcomes:

Statistically significant differences in physical activity were detected between IL and SG groups at 3 months $(\beta=-0.91$ $(\mathrm{se}=0.458), 95 \% \mathrm{CI}(-1.81,-0.004), p=0.048)$, with the IL group having higher PA activity levels compared to the SG group. No other significant differences were found in PA at 12 months. No statistically significant differences were detected between groups at any time point with respect to the weight variable.

Secondary outcomes:

No statistically significant differences were detected between groups at any time point with BMI or waist variables. Statistically significant differences were detected between IL and SG groups at 12 months only in SBP $(\beta=7.87(\mathrm{se}=3.17)$, $95 \% \mathrm{CI}(1.59,14.15), p=0.014)$ and DBP $(\beta=5.23(\mathrm{se}=1.82)$, $95 \%$ CI $(1.61,8.84), p=0.004)$, with women in the IL group having smaller increases in blood pressure.

\section{DISCUSSION}

We found women who participated in a high-contact, interventionist-led physical activity and weight loss intervention were able to successfully increase their physical activity levels in the short-term (3 months). The increases in the IL group were comparable to those seen in the Diabetes Prevention Program and roughly equivalent to adding $90 \mathrm{~min}$ of walking at moderate pace per week. The increases in the IL group were greater than those in the SG group at 3 months shortly after the conclusion of the intervention; the difference between groups was no longer significant at 12 months. Women in both groups experienced modest decreases in weight, BMI and waist circumference at both 3 and 12 months, but the differences between the groups were not significant at either time point.

A recent systematic review and meta-analysis found that promotion of physical activity to sedentary adults recruited in primary care resulted in significant increases in physical activity levels at 12 months. ${ }^{28}$ The majority of the 15 included studies had both female and male participants, with the exception of one with only women and one with only men. The women only study included over 1,000 women recruited from 17 primary care clinics in New Zealand and demonstrated increased physical activity and quality of life over two years. ${ }^{29}$ One focus of the meta-analysis was to compare interventions primarily conducted in the primary care setting and by the primary care providers with those that were "referral schemes" that sent patients elsewhere (e.g., community centers) to receive an intervention. Only three of the 15 included studies were referral schemes and the authors concluded that these interventions were not superior to other interventions based in primary care settings that might be lower in cost. Our intervention was a hybrid of those discussed in the meta-analysis, as it was conducted in the primary care setting rather than a separate location or facility, but delivered by staff other than the participants' usual providers.

Reviews of weight interventions conducted in primary care settings have concluded that only high-intensity interventions are effective in promoting weight loss. ${ }^{9}$ The amount of weight lost by our participants was very modest, and lower than that reported by other primary care weight loss interventions. We believe that is likely due to the fact that while our intervention was high-contact, we focused more on physical activity than on diet behavior. Given that physical activity may be more successful at maintaining than inducing weight loss, ${ }^{30,31}$ it is not surprising that we saw only a very modest and nonsignificant amount of weight loss in our study.

Our study had several strengths. We successfully recruited a racially diverse group of women. The proportion of Black women included in our study exceeded that of any study included in the recent meta-analysis, many of which were conducted in European populations. Our race-stratified analyses showed a similar intervention effect at 3 months, although the result was not statistically significant in non-white women, likely due to the fact that our study was not powered to detect differences by race. Our study also included a novel mindfulness component. In a 2008 JAMA Commentary, Ludwig and Kabat-Zinn discussed the relationship between mindfulness and medicine, suggesting that mindfulness could promote a more participatory medicine through an increase in selfresponsibility for one's life choices, including modifiable lifestyle factors that often create or exacerbate chronic medical conditions. ${ }^{19}$ They also noted that mindfulness may influence an individual's motivation for lifestyle changes involving diet, physical activity, smoking cessation, or other behaviors. ${ }^{19}$ Several mindfulness studies have demonstrated an increase in exercise or physical activity behaviors as a secondary outcome. ${ }^{32,33}$

Our study had limitations. Our follow-up for women in the self-guided intervention was lower than desired at the 3-month time point, although we did improve at 12 months by adding a financial incentive. We used some data collected by phone at 12 months, which included self-reported weight data that may be biased by under-report. Our intervention was delivered by highly trained interventionists, which may limit its generalizability to other primary care settings, as does the fact that it was conducted in an academic setting in one city. Given that the IL intervention covered many topics and included both 
discussion and physical activity, it is difficult to know which portion of the IL intervention was most related to behavior change. Lastly, even though we used an intervieweradministered and well-validated physical activity measure, the MAQ is a self-report measure and may be subject to recall bias.

In conclusion, we were able to show a significant increase in short-term physical activity levels in a group of previously sedentary women who are representative of a primary care population. Future efforts should focus on determining the best way to sustain increases in physical activity using resources available in primary care settings.

Acknowledgements: Healthy Bodies, Healthy Hearts was supported by a K23 grant to Dr. Conroy from the National Institutes of Health, National Heart, Lung and Blood Institute (K23 HL 085405-02).

Conflict of Interest: The authors declare that they do not have a conflict of interest.

Corresponding Author: Molly B. Conroy, MD, MPH; University of Pittsburgh, Pittsburgh, PA, USA (e-mail: conroymb@upmc.edu).

\section{REFERENCES}

1. Ogden CL, Carroll MD, Kit BK, Flegal KM. Prevalence of childhood and adult obesity in the United States, 2011-2012. JAMA. 2014;311(8):806-14.

2. Flegal KM, Graubard BI, Williamson DF, Gail MH. Cause-specific excess deaths associated with underweight, overweight, and obesity. JAMA. 2007;298(17):2028-37.

3. Tucker JM, Welk GJ, Beyler NK. Physical activity in U.S.: adults compliance with the Physical Activity Guidelines for Americans. Am J Prev Med. 2011;40(4):454-61.

4. Ogden CL, Carroll MD, Curtin LR, McDowell MA, Tabak CJ, Flegal KM. Prevalence of overweight and obesity in the United States, 1999-2004. JAMA. 2006;295(13):1549-55.

5. Muennig P, Lubetkin E, Jia H, Franks P. Gender and the burden of disease attributable to obesity. Am J Public Health. 2006;96(9):1662-8. PMCID: PMC1551950.

6. Bertakis KD, Azari R, Helms LJ, Callahan EJ, Robbins JA. Gender differences in the utilization of health care services. J Fam Pract. 2000;49(2):147-52.

7. USDHHS. Physical Activity Guidelines Advisory Committee final report.; [cited]; Available from: www.health.gov/PAGuidelines/Report/ Default.aspx.

8. Behavioral counseling in primary care to promote physical activity: recommendations and rationale. Am Fam Physician. 2002;66(10): 1931-6.

9. Leblanc ES, O'Connor E, Whitlock EP, Patnode CD, Kapka T. Effectiveness of primary care-relevant treatments for obesity in adults: a systematic evidence review for the U.S. Preventive Services Task Force. Ann Intern Med. 2011;155(7):434-47.

10. Lemon SC, Rosal MC, Zapka J, Borg A, Andersen V. Contributions of weight perceptions to weight loss attempts: differences by body mass index and gender. Body Image. 2009;6(2):90-6. PMCID: PMC2692706.
11. Centers for Disease C, Prevention. Trends in leisure-time physical inactivity by age, sex, and race/ethnicity-United States, 1994-2004. MMWR Morb Mortal Wkly Rep. 2005;54(39):991-4.

12. Matthews KA, Meilahn E, Kuller LH, Kelsey SF, Caggiula AW, Wing RR. Menopause and risk factors for coronary heart disease. N Engl $J$ Med. 1989;321(10):641-6.

13. Kuller LH, Kriska AM, Kinzel LS, Simkin-Silverman LR, Sutton-Tyrrell $\mathbf{K}$, Johnson BD, et al. The clinical trial of Women on the Move through Activity and Nutrition (WOMAN) study. Contemp Clin Trials. 2007;28(4):370-81. PMCID: PMC1941838.

14. Kuller LH, Pettee Gabriel KK, Kinzel LS, Underwood DA, Conroy MB, Chang Y, et al. The Women on the Move Through Activity and Nutrition (WOMAN) study: final 48-month results. Obesity (Silver Spring). 2012;20(3):636-43. PMCID: PMC3623568.

15. Kriska AM, Caspersen CJ. Introduction to a collection of physical activity questionnaires. Med Sci Sports Exerc. 29:S5-9.

16. Kuller LH, Simkin-Silverman LR, Wing RR, Meilahn EN, Ives DG. Women's Healthy Lifestyle Project: a randomized clinical trial: results at 54 months. Circulation. 2001;103(1):32-7.

17. Simkin-Silverman LR, Wing RR, Boraz MA, Kuller LH. Lifestyle intervention can prevent weight gain during menopause: results from a 5-year randomized clinical trial. Ann Behav Med. 2003;26(3):212-20.

18. Knowler WC, Barrett-Connor E, Fowler SE, Hamman RF, Lachin JM, Walker EA, et al. Reduction in the incidence of type 2 diabetes with lifestyle intervention or metformin. N Engl J Med. 2002;346(6):393-403. PMCID: PMC1370926.

19. Ludwig DS, Kabat-Zinn J. Mindfulness in medicine. JAMA. 2008;300(11):1350-2

20. Koffman DM, Bazzarre T, Mosca L, Redberg R, Schmid T, Wattigney WA. An Evaluation of Choose to Move 1999: an American Heart Association Physical Activity Program for Women. Arch Intern Med. 2001;161(18):2193-9.

21. Kendzierski D, DeCarol KJ. Physical Activity Enjoyment Scale: two validation studies. J Sport Exerc Psychol. 1991;13:50-64.

22. Beck AT, Ward CH, Mendelson M, Mock J, Erbaugh J. An inventory for measuring depression. Arch Gen Psychiatry. 1961;4:561-71.

23. Buysse DJ, Reynolds CF 3rd, Monk TH, Berman SR, Kupfer DJ. The Pittsburgh Sleep Quality Index: a new instrument for psychiatric practice and research. Psychiatry Res. 1989;28(2):193-213.

24. Ware JE Jr, Sherbourne CD. The MOS 36-item short-form health survey (SF-36). I. Conceptual framework and item selection. Med Care. 1992;30(6):473-83.

25. Marcus BH, Dubbert PM, Forsyth LH, McKenzie TL, Stone EJ, Dunn AL, et al. Physical activity behavior change: issues in adoption and maintenance. Health Psychol. 2000;19(1 Suppl):32-41.

26. Marcus BH, Rossi JS, Selby VC, Niaura RS, Abrams DB. The stages and processes of exercise adoption and maintenance in a worksite sample. Health Psychol. 1992;11(6):386-95.

27. Marcus BH, Selby VC, Niaura RS, Rossi JS. Self-efficacy and the stages of exercise behavior change. Res Q Exerc Sport. 1992;63(1):60-6.

28. Orrow G, Kinmonth A-L, Sanderson S, Sutton S. Effectiveness of physical activity promotion based in primary care: systematic review and meta-analysis of randomised controlled trials. BMJ. 2012;344.

29. Lawton BA, Rose SB, Elley CR, Dowell AC, Fenton A, Moyes SA. Exercise on prescription for women aged 40-74 recruited through primary care: two year randomised controlled trial. BMJ. 2008;337:a2509. PMCID: 2769033.

30. Lee IM, Djousse L, Sesso HD, Wang L, Buring JE. Physical activity and weight gain prevention. JAMA. 2010;303(12):1173-9. PMCID: PMC2846540.

31. Jakicic JM. The role of physical activity in prevention and treatment of body weight gain in adults. J Nutr. 2002;132(12):3826S-9S.

32. Carlson LE, Speca M, Patel KD, Goodey E. Mindfulness-based stress reduction in relation to quality of life, mood, symptoms of stress and levels of cortisol, dehydroepiandrosterone sulfate (DHEAS) and melatonin in breast and prostate cancer outpatients. Psychoneuroendocrinology. 2004;29(4):448-74.

33. Roth B, Creaser T. Mindfulness meditation-based stress reduction: experience with a bilingual inner-city program. Nurse Pract. 1997;22(3):150-2. 4, 7 passim. 